\title{
El cuerpo en la Cultura Visual a través del videoclip Fuego de Vetusta Morla
} The body in Visual Culture through the video clip Fuego de Vetusta Morla

Carmen Flores Hurtado

Universidad de Málaga (cfloresh24@uma.es)

RESUMEN: El videoclip musical supone uno de los medios más potentes dentro de la Cultura Visual. Su relación con otras artes le lleva a conformarse como una forma de expresión híbrida, donde los aspectos cinematográficos, el sonido y la luz juegan un papel fundamental. El cuerpo, como uno de los elementos clave de la Historia del Arte, tomará un gran protagonismo dentro del mundo audiovisual, convirtiéndose en el principal protagonista, en muchos casos, de multitud de disciplinas y formas de expresión artística. A través del videoclip Fuego de la banda Vetusta Morla se podrán estudiar distintos aspectos de las relaciones entre el cuerpo y la Cultura Visual.

PALABRAS CLAVE: Videoclip musical, Cultura Visual, Cuerpo, Disciplina artística, Hibridación, Vetusta Morla.

\begin{abstract}
The music video is one of the most powerful media in the Visual culture. His relationship with other arts leads him to be formed as a form of hybrid expression, where cinematographics aspects, sound and light play a fundamental role. The body, as one of the key elements in the Art History, will take a great role in the audiovisual world, becoming the main protagonist, in many cases, of a multitude of disciplines and forms of artistic expression. Through the video Fuego of the group Vetusta Morla will be able to study different aspects of the relationship between the body and Visual culture.
\end{abstract}

KEYWORDS: Music video, Visual culture, Body, Artistic discipline, Hibridation, Vetusta Morla. 
El videoclip, entendido como elemento de la Cultura Visual, ha experimentado diversos cambios desde su aparición alrededor de la segunda mitad del siglo pasado. Se debe entender como una forma de expresión, como un medio que conecta con la cultura musical, pero también como un elemento comercial, pues, a fin de cuentas, sirve para incrementar las ventas de discos. En este artículo se dejará a un lado la vertiente de marketing para centrarse en la visual, a partir de un videoclip musical del grupo Vetusta Morla, en referencia a su tema Fuego. El objetivo principal es el de dar constancia de la importancia que tiene el cuerpo humano en el desarrollo actual de la Cultura Visual a través de diversos ejemplos. Por tanto, se hará un recorrido breve por la historia del videoclip, para después hablar de la importancia del cuerpo humano dentro de éste y, en concreto, de los aspectos iconográficos que aguarda el videoclip a tratar.

\section{Breves apuntes sobre el Videoclip y su desarrollo}

El videoclip musical está ligado a multitud de artes, produciéndose una hibridación de las disciplinas que viene a ser muy frecuente desde mediados del siglo XX. Su relación con otras formas de expresión como la música y la danza le llevan a ser uno de los elementos más potentes dentro de la Cultura Visual. El cuerpo es también un elemento señero que puede ser estudiado desde la perspectiva de casi cualquier disciplina, aunque es cierto que el videoclip es muy propicio para ello por ser una fuente documental y visual.

Hay que decir que, aunque fue entre los años setenta y ochenta cuando se produjo el boom del videoclip, se pueden citar como antecedentes las películas que se realizaban en los años veinte para acompañar en los conciertos de jazz (Sedeño, 2002: 1). Todo esto se materializó aún más con el cine hollywoodiense, donde la música tomó mucho protagonismo, convirtiendo las películas en elementos teatralizados y en musicales a través de la pantalla.

A partir de los años sesenta, con la llegada de estilos como el rock and roll o el blues, los artistas comenzaron a tener la necesidad de comercializar sus trabajos yendo un paso más allá; como el caso de Los Beatles, que decidieron unir imagen y música para Strawberry Fields Forever (1967) (Oliveira, 2009: 8), aunque no fueron los únicos que hicieron esto, ya que otro grupo histórico como Queen lo hizo también para su videoclip más emblemático, Bohemian Rhapsody (1975), que, además, visualmente, se asemeja al que se tratará más adelante. 
Un paso más se dio ya en la década de los ochenta, donde primaban el hard rock y el glam metal ante otros estilos, y donde grupos como Whitesnake, Def Leppard ${ }^{1}$ o Scorpions, saltaron a la fama con sus videoclips; en el caso de los primeros, se podría destacar el videoclip del tema Is this love? (1987), donde ya se puede ver un salto en comparación con, por ejemplo, el mencionado de Los Beatles. Otro gran videoclip de la época que hay que mencionar, dentro del terreno del pop, es el de Thriller (1982) de Michael Jackson, cargado de una estética muy cinematográfica.

Sin duda alguna, el videoclip, desde su posición dentro de la Cultura Visual como un medio de gran potencia, actúa también como objeto de reflexión sobre la construcción de los nuevos procesos culturales (Sedeño, 2012: 93). Hay que mencionar que los videoclips no podrían entenderse sin el cine, uno de sus antecedentes más claros, del cual ha tomado la estética, los planos, la narrativa.; también de otras artes como la pintura, habiéndose realizado clips de vídeo muy inspirados en grandes obras de la Historia del Arte².

\section{El cuerpo humano: elemento transmisor en el Videoclip}

Dentro del ámbito del videoclip son frecuentes los usos de simbologías e iconografías que llevan al espectador más allá del propio tema musical. Uno de los aspectos que más se ha representado a lo largo de la Historia del Arte, y también presente en la Cultura Visual, es el cuerpo, entendido como medio de expresión y reflexión, aunque también como elemento de provocación o insinuación.

La posición del cuerpo dentro de todo lo que conforma la Cultura Visual también ha cambiado, y ha ido convirtiéndose en una nueva forma de lenguaje, en una narrativa. Con las prácticas performativas el cuerpo experimentó su máximo apogeo, pues era el contenido y continente de la obra de arte al mismo tiempo. Las artes de acción pusieron en valor, más que nunca, el potencial que guardaba el ser humano en sí mismo. Las artes como el cine fueron desarrollando herramientas para dotar de más importancia al cuerpo, con el uso de algunas técnicas como el primer plano (Sedeño, 2012: 96), que comenzó a tener gran relevancia por la desjerarquización de la figura y el detallismo de ésta, al mismo tiempo.

\footnotetext{
${ }^{1}$ Del grupo Def Leppard podemos destacar el videoclip de Loves Bites del año 1987, donde también aparece el cuerpo como protagonista; en este caso femenino, como en la mayoría de los videoclips de hard rock de la época, sobre todo en las baladas de este estilo.

2 Como el caso del videoclip de Coldplay del tema Viva la vida (2008), inspirado en la obra La libertad guiando al pueblo (1830) de Delacroix.
} 
Es habitual encontrar videoclips donde el cuerpo humano tome el protagonismo y en donde aparezca para expresar algo. El cuerpo actúa descontextualizado, en un nuevo entorno donde es desmembrado para transmitir un mensaje diferente al de la vida diaria. Esta nueva concepción en los medios audiovisuales puede tomar sus inicios en la performance, donde el cuerpo invade un espacio para transformarlo con algún objetivo determinado. En el caso del videoclip, actúa como medio transmisor de ideas, como agente transformador a través de una pantalla que conecta con el espectador y que le invita a percibir ciertas sensaciones. Se convierte en un objeto cultural, adquiriendo también la función de sujeto, sobre todo en el ámbito audiovisual.

A lo largo de la Historia del Arte, la representación del cuerpo ha ido variando, transmitiendo distintos mensajes a medida que se daban diferentes situaciones sociales y políticas, cambiando su canon, transformándose; en definitiva, ha ido mutando hasta llegar a ser lo que es hoy. A partir de la mitad del siglo XX, el cuerpo se convertirá en objeto principal para las nuevas formas de creación artística de la época, donde servirá como molde de trabajo, actuando sobre el mismo para llevar a cabo determinadas acciones.

A medida que estas nuevas formas de creación se van desarrollando, se irá indagando también cada vez más en los aspectos relacionados con el cuerpo humano, intentando llegar a conocer sus límites y significados, además de la propia iconografía que esconde, con el fin de que el ser sea conocedor de sí mismo. En el caso del videoclip, será frecuente que el mismo artista se convierta en el principal intérprete de la acción, mientras que a la vez involucrará al espectador. El cuerpo ahora ha cambiado su canon, alejándose de la concepción de lo bello y pensando más en un modo de construcción social, en un arma de transmisión directa a la sociedad; se ha convertido en un objeto artístico capaz de poner a prueba los límites de la tolerancia social, provocando y generando preguntas en ese espectador que está mirando (Adrián, 2007: 153).

El videoclip que concierne en este artículo, donde se trata el tema del cuerpo, pertenece a Vetusta Morla, una banda originada en Tres Cantos (Madrid) en $1998^{3}$. Sus videoclips son creados con una fuerte simbología y con potentes mensajes, como también sus letras, pues siempre hacen reflexionar a sus seguidores.

Este tema contiene un mensaje social más que político, y está inspirado en una historia que le fue contada al guitarrista rítmico y principal compositor de la banda, Guillermo Galván. Aunque el

\footnotetext{
${ }^{3}$ Vetusta Morla es considerada una de las bandas más populares de España, sobre todo dentro del plano del rock y del movimiento independiente, más conocido como indie.
} 
videoclip representa otros aspectos más subjetivos, la anécdota citada se refiere a la situación que vivió un pueblo indígena al que, en una visita, le llevaron ropas para que vistieran, las cuales, tras el contacto con su piel, les produjeron gripes y diversas enfermedades. Con esto se quiere transmitir la idea de que la imposición no siempre es buena, ni tampoco el progreso. Ese pueblo, por querer ser modernizado, sufrió graves consecuencias. Se puede equiparar a todos los aspectos de la vida, a la importancia de no imponer las ideas o actos sobre las personas, pues no siempre da buen resultado.

Se pretende transmitir la idea de lo erróneo que resulta querer cambiar las cosas de manera tan radical, cuando realmente lo básico y fundamental habita dentro de nosotros mismos, los que tenemos la fuerza para llevarlo todo a cabo. Si pretendemos cambiar nuestro destino, no siempre va a salir bien, como el caso que hemos mencionado.

Durante todo el tiempo que dura el videoclip, lo que transmite el cuerpo que aparece va en relación con la letra que es cantada. Los gestos y movimientos hablan por sí solos. No siempre ocurre en los videoclips musicales, pues en muchas ocasiones las personas que aparecen, y con ellas sus propios cuerpos, simplemente actúan como si de un corto fílmico se tratara; no realizan ninguna función que vaya en consonancia con la letra, simplemente adornan el videoclip, son un elemento más. En este caso, en el vídeo de Fuego, el único protagonista es el cuerpo, y por ello tiene esa fuerte carga simbólica.

El videoclip comienza mostrando un montón de ropa que se vislumbra entre la oscuridad del fondo mientras suena la música inicial del tema. La canción Fuego comienza diciendo «Trajeron ropas para impresionar, trapos y pieles en forma de abrigo. Pero ¿quién quiere taparse si aún no conoceel frío?»y, a partir de este momento, la masa de telas se convierte en un cuerpo que se va irguiendo poco a poco. Se ve esa idea en la que está inspirada el videoclip; la idea equivocada de progresión.

A medida que se desarrollan las siguientes estrofas, el cuerpo se va liberando. El intérprete, en este caso el vocalista de la banda, actúa como un auténtico performer que no para de realizar su acción hasta que finaliza la canción. Sus movimientos y su forma de transmitir los mensajes de las letras son una muestra clara de la importancia que el cuerpo tiene en la Cultura Visual y de cómo puede hablar por uno mismo con unos simples gestos.

La parte más importante de la canción y del videoclip viene con el estribillo: «Fuego, siempre alrededor. Fuego, alguien olvidó que el fuego, que el fuego... lo guardo yo»; en este momento el cuerpo se refugia en sí mismo para protegerse, pero poco a poco se vuelve a erguir dándose cuenta de la verdadera fuerza que guarda. Se vuelve a levantar y se sigue liberando de sus ataduras. 
Es importante señalar cómo en la canción se refieren al fuego como un arma, como la verdadera fuerza de uno mismo. Todo esto lo va transmitiendo el cuerpo de principio a fin; va recuperando el aliento y las ganas y se hace una muy correcta expresión del mensaje principal. El ser humano, teniendo el fuego, tiene la capacidad de filosofar; esto se podría comparar con El mito de la caverna y la idea del fuego como luz del conocimiento frente a la ignorancia, pero un conocimiento que debe ser controlado, pues de lo contrario, podríamos quemarnos.

Todas estas ideas se materializan en un videoclip muy minimalista en cuanto a estética, pero con una fuerte carga emocional donde predominan tres elementos clave: el movimiento, el cuerpo y la luz. Sobre un fondo negro que remite al ámbito cinematográfico, se posiciona una figura humana sobre un pedestal que no deja de girar durante todo el videoclip, exponiéndose ante el espectador como si de una escultura se tratase; está ahí para ser observado. El movimiento es un elemento muy señero, pues de principio a fin estará presente, mostrando todas las caras del intérprete y la evolución que sufre a lo largo del vídeo. Se tornará a veces más rápido y otras cambiará de dirección, haciendo referencia también a las complejidades del ser y a sus distintas facetas.

El cuerpo es el verdadero protagonista del videoclip ${ }^{4}$. Al inicio del tema, aparece un plano general que es acompañado por los primeros acordes; al fondo, casi en penumbra, una masa agachada que deja intuir ciertas texturas de ropajes [1]. Del general se pasa a un plano principal, donde, a medida que se empiezan a escuchar las primeras palabras de la canción, una forma humana comienza a moverse entre todo ese montón de telas; un cuerpo que representa al ser invadido que quiere despojarse de aquello innecesario y superfluo que le ha sido impuesto. La anatomía masculina se empieza a apreciar entre las luces y sombras.

A medida que avanza el tema, el cuerpo comienza a erguirse, tomando cada vez más fuerza, con movimientos en crescendo al igual que la letra y el instrumental. Se va despojando de esos ropajes que le tenían oprimido. Con movimientos muy teatralizados, la tela va cayendo hacia la plataforma donde se sitúa Pucho, el intérprete de la coreografía. La forma de moverse recuerda a la danza contemporánea; es una performance, un acto efímero que queda materializado en formato vídeo.

\footnotetext{
${ }^{4}$ La presencia del cuerpo de la manera vista en Fuego, como medio expresivo, se ha podido ver en otros videoclips como Somebody that I used to know (2011) de Gotye o Pagan Poetry (2012) de Björk.
} 


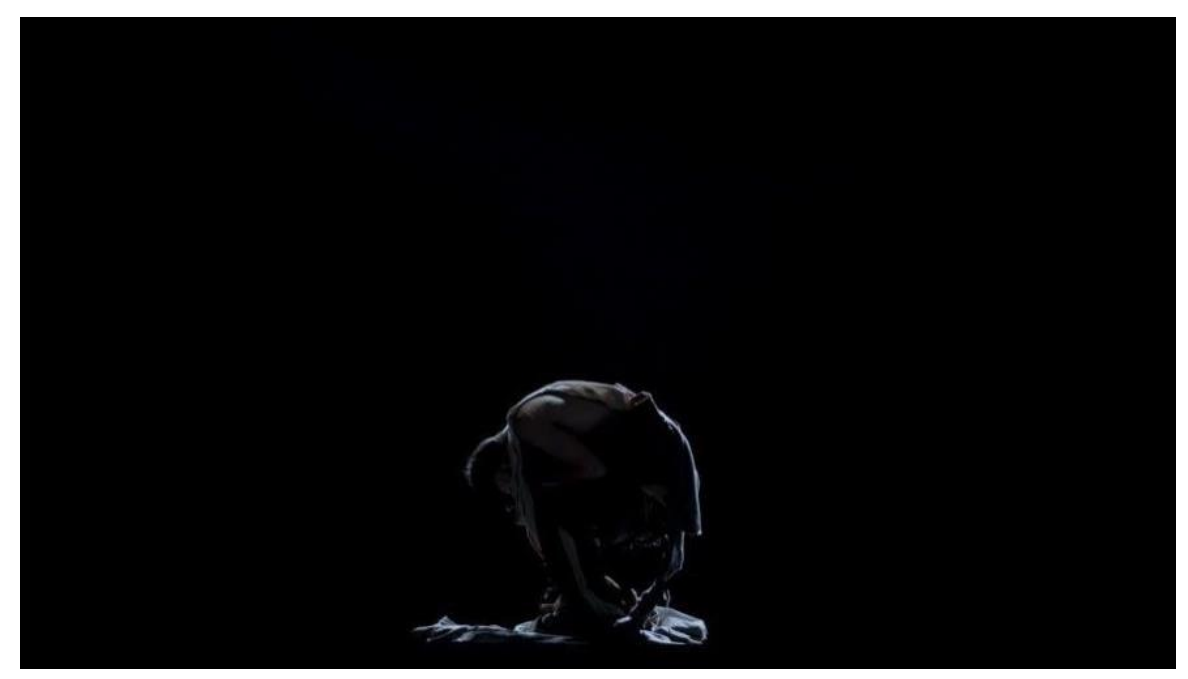

1. Imagen del videoclip donde se ve al cuerpo cubierto por las telas, casi en penumbra. Fuente: Paperblog.

El cuerpo, que ya está mucho más liberado que al comienzo, va realizando cada vez una acción con más ímpetu, con movimientos desgarradores y otros llenos de significados. Se aprecia un momento en que la figura, erguida, apunta con el dedo índice hacia arriba [2], aunque mira al suelo, como una especie de llamamiento -y a la vez subordinación- a una fuerza superior que está ahí arriba; tiene la necesidad de aferrarse a algo. Aparece con el torso cubierto por una tela a modo de banda, que recuerda a las representaciones en la Historia del Arte de San Juan Bautista, o incluso a la representación de Platón en La Escuela de Atenas (1510-1512) de Rafael.

A medida que avanza el clip, el cuerpo seguirá realizando movimientos en referencia a la letra, como en el momento en que se canta «¿Quién quiere alarmas si aún sigo vivo?», y el artista flexiona su cuerpo en actitud de clamar hacia la fuerza superior mencionada ${ }^{5}$, gritándole que sigue vivo, que está ahí y que sigue con fuerzas [3]. Por otra parte, el único momento en que se ve al intérprete cantar será en el estribillo final, cuando con más fuerza que en todo el tema, grita: «ique el fuego lo guardo yo!». En ese momento, el cuerpo no está apresado como al inicio, que se hundía entre las telas sin capacidad para salir ni levantarse; ahora es poderoso y más expresivo; imparable. Por último, se concluye con el ser liberado por completo, sin telas ni ataduras encima, mostrando su anatomía aún con contrastes, y con una postura totalmente erguida, habiéndose ya recuperado.

5 Podemos decir que el cuerpo clama al cielo para significarse y decir que está más vivo que nunca. 


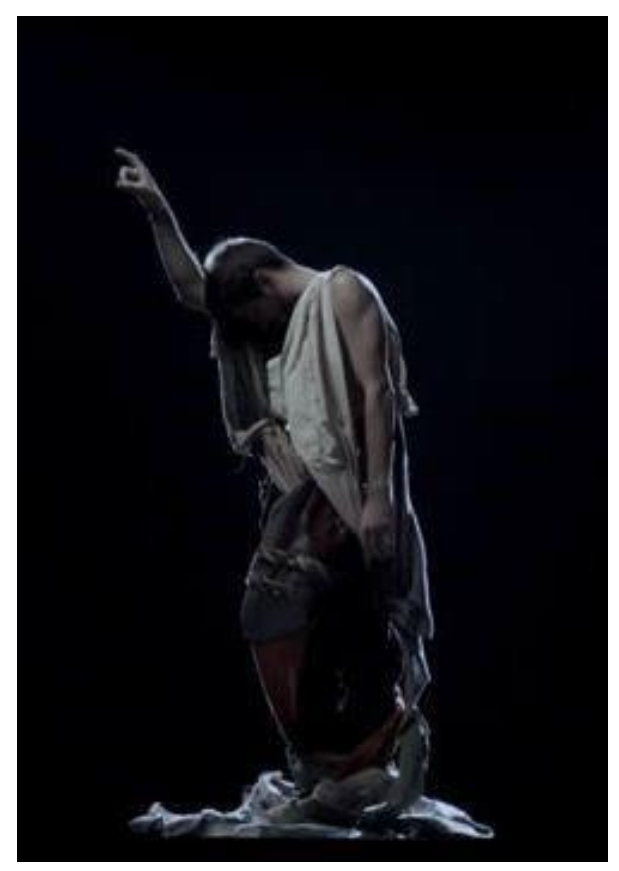

2. El intérprete del videoclip apuntando hacia arriba. Fuente: Google

Durante todo el videoclip se aprecia la transición que va sufriendo el cuerpo; cómo pasa de un estado a otro: del aprisionamiento a la libertad. El intérprete, al que se puede considerar performer, expresa a través de sus propios movimientos todos los mensajes que aguarda la canción que está siendo interpretada.

Aparte del cuerpo, se ha mencionado también la importante presencia de la luz como elemento dentro del videoclip. Desde el inicio adquiere un gran protagonismo, actuando como moldeadora de la figura en todo momento. Se crean claroscuros que hacen que el cuerpo se distinga mínimamente al inicio, dejando entrever las ropas y algunos signos de la anatomía. Pero, a medida que avanza la canción, la luz tomará más relevancia, haciendo acto de presencia en momentos determinados, aunque sin abandonar ese contraluz que recuerda a la pintura barroca y a todas esas obras de artistas como Caravaggio donde a menudo las figuras no se distinguen del todo, pero sin embargo el tratamiento de las telas es impecable. Aquí ocurre algo parecido; y es que, aunque el cuerpo sea el protagonista del videoclip, el hecho de que la luz le moldee nos hace visualizarlo desde distintos planos, sin permitir que sea visto por completo en ningún momento. 


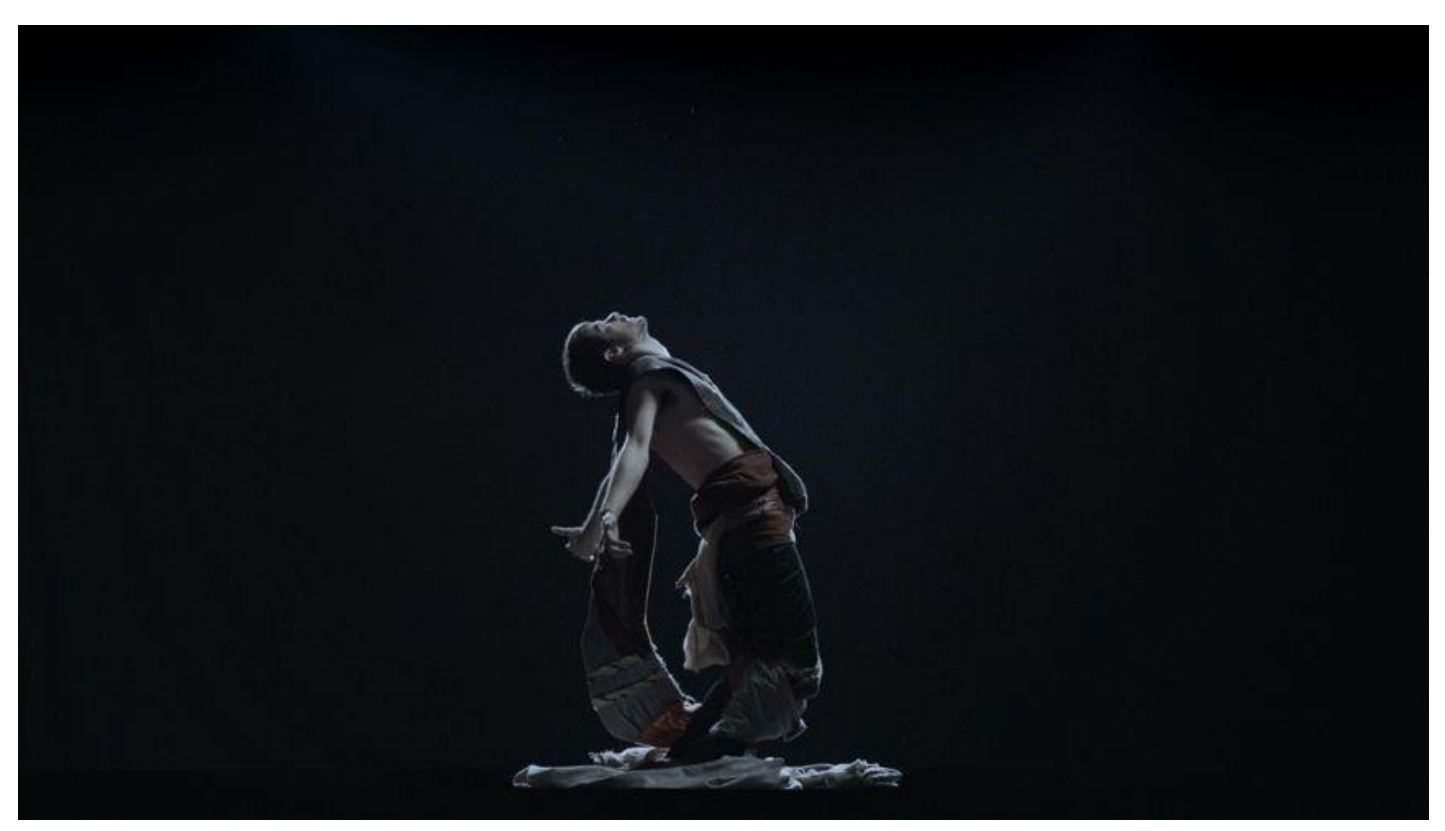

3. Captura del videoclip donde se aprecia ese clamor al cielo. Fuente. YouTube.

En un plano más iconográfico, y aunque la luz sea considerada como un elemento divino, se puede interpretar aquí como signo de «verdad» ${ }^{6}$, basándose en el contexto laico en que se elabora el videoclip. Esa verdad es contada y expresada por el cuerpo, haciendo referencia a esas ataduras del ser, a esa liberación compleja y necesaria. La luz va a tener su propio discurso junto a la figura humana, va a hablar al espectador. En uno de los momentos, penetrará casi por completo en el cuerpo, cuando se canta la estrofa antes mencionada: «¿Quién quiere alarmas si aún sigo vivo?», cuando la única opción posible ya es clamar, y la luz impacta como símbolo de presencia y de vida, de verdad absoluta $^{15}$; un foco minimalista pero más poderoso que en todo el videoclip, símbolo esclarecedor ${ }^{7}$.

Al igual que se le ha dado importancia a la luz, sería conveniente dársela también a las sombras, ya que adquieren un gran protagonismo en el desarrollo del videoclip, permitiendo ver durante todo el proceso esa dualidad con la luz; esa diferencia entre la verdad y el lado oscuro, que conformarán un diálogo pudiéndose relacionar con lo interno de cada uno. La búsqueda de la verdad, a través de la luz, y el hundimiento en las sombras, como se encuentra el cuerpo al comienzo del videoclip. Las sombras

\footnotetext{
6 A pesar de esto, en el contexto de los medios audiovisuales la luz actúa también de una forma teatralizada. En el caso que nos ocupa, podemos afirmarlo.

${ }^{7}$ La presencia de ese foco potente de luz sí es más aplicable al ámbito religioso. Esto se ve en otros videoclips como el de Losing my Religion (1991) de R.E.M., donde también aparece esta misma simbología. En el videoclip de Fuego, sin embargo, se puede interpretar como la verdad nuevamente.
} 
también moldean el contorno de la figura, mostrando sus dos caras desde una mirada caleidoscópica. A pesar de todo, será la luz la que triunfe frente a las sombras.

\section{Conclusiones}

Tras haber hecho un análisis por el videoclip Fuego ${ }^{8}$ de Vetusta Morla se llega a la conclusión de la importancia que tiene el cuerpo en casos como este. Cómo se convierte en protagonista tomando a veces más relevancia que la propia letra de la canción, pues consigue hablar por sí solo. Con una cuidada coreografía, en este videoclip el cuerpo es un elemento primordial que da mucho más sentido a los mensajes que se quieren transmitir.

El videoclip tratado aguarda multitud de iconografías que derivan en una especie de trampantojo que lleva a nombrar el término «neobarroco», no haciendo referencia a la vuelta del barroco como estilo, sino a una nueva forma de ver la realidad, de manera deformada y desde varios puntos de vista 9 .

Se queda constancia de que el videoclip es un formato de vital importancia para tratar el tema del cuerpo humano y mirarlo desde otras perspectivas más alejadas de las artes tradicionales.

\section{Referencias bibliográficas}

ARRANZ RASO, Paula, «El cuerpo. Una visión a través del arte». En: <https://goo.gl/88tQ6Q> (Fecha de consulta: 21-08-2018).

KORNDORFFER VELASCO, Susana, «Del cuerpo como objeto del arte, al cuerpo como arte-objeto». En: 〈http://fepal.org/wp-content/uploads/314-esp.pdf> (Fecha de consulta: 18-06-2018).

LÓPEZ SILVESTRE, Federico (2004), El paisaje virtual: el cine de Hollywood y el neobarroco digital, Biblioteca Nueva, Madrid.

\footnotetext{
${ }^{8}$ El videoclip ha sido dirigido por Fernando Franco, sobre una idea de Guillermo Galván, con la coreografía de Antonio Ruz y la interpretación de Pucho (2014).

${ }^{9}$ Una idea parecida sostiene Federico López Silvestre en su libro El paisaje virtual. El cine de Hollywood y el neobarroco digital, donde analiza las nuevas perspectivas del término «neobarroco» aplicándolo al ámbito cinematográficoa través de películas como Matrix o Star Trek.
} 
OLIVEIRA DE ARAÚJO, Taize (2009), Videoclip y cuerpo: el entre-lugar de los corpus mutantes, Universidad Autónoma de Barcelona, Barcelona. p. 8.

SEDEÑO VALDELLÓS, Ana (2002), «Música e imagen: aproximación a la historia del vídeo musical», Área Abierta, no 3, pp. 1-9.

SEDEÑO VALDELLÓS, Ana (2012), «Video musical y cultura: propuestas para analizar el cuerpo en el videoclip», Revista de Comunicación Vivat Academia, nº 120, p. 93.

Videoclip de Fuego: <https://www.youtube.com/watch?v=H3ZCKXX7tVI> (Fecha de consulta: 2006-2018). 